Kragujevac Journal of Mathematics

Volume 45(4) (2021), Pages 587-596.

\title{
EXISTENCE OF POSITIVE SOLUTIONS FOR A CLASS OF KIRRCHOFF ELLIPTIC SYSTEMS WITH RIGHT HAND SIDE DEFINED AS A MULTIPLICATION OF TWO SEPARATE FUNCTIONS
}

\author{
YOUCEF BOUIZEM $^{1}$, SALAH BOULAARAS $^{2,3}$, AND BACHIR DJEBBAR $^{1}$
}

ABSTRACT. The paper deals with the study of existence of weak positive solutions for a new class of Kirrchoff elliptic systems in bounded domains with multiple parameters, where the right hand side defined as a multiplication of two separate functions.

\section{INTRODUCTION}

In this paper, we consider the following system of differential equations

$$
\left\{\begin{array}{l}
-A\left(\int_{\Omega}|\nabla u|^{2} d x\right) \triangle u=\lambda_{1} \alpha(x) f(v) h(u) \text { in } \Omega, \\
-B\left(\int_{\Omega}|\nabla v|^{2} d x\right) \triangle v=\lambda_{2} \beta(x) g(u) \tau(v) \text { in } \Omega, \\
u=v=0 \text { on } \partial \Omega,
\end{array}\right.
$$

where $\Omega \subset \mathbb{R}^{N}, N \geq 3$, is a bounded smooth domain with $C^{2}$ boundary $\partial \Omega$, and $A, B: \mathbb{R}^{+} \rightarrow \mathbb{R}^{+}$are continuous functions, $\alpha, \beta \in C(\bar{\Omega}), \lambda_{1}$ and $\lambda_{2}$ are nonnegative parameters.

Since the first equation in (1.1) contains an integral over $\Omega$, it is no longer a pointwise identity, therefore, it is often called nonlocal problem. This problem models several physical and biological systems, where $u$ describes a process which depends

\footnotetext{
Key words and phrases. Kirrchoff elliptic systems, existence, positive solutions, sub-supersolution, multiple parameters

2010 Mathematics Subject Classification. Primary: 35J60. Secondary: 35B30 35B40.

DOI 10.46793/KgJMat2104.587B

Received: November 04, 2018.

Accepted: March 18, 2019.
} 
on the average of itself, such as the population density, see [9]. Moreover, problem (1.1) is related to the stationary version of the Kirchhoff equation

$$
\rho \frac{\partial^{2} u}{\partial t^{2}}-\left(\frac{P_{0}}{h}+\frac{E}{2 L} \int_{0}^{L}\left|\frac{\partial u}{\partial x}\right|^{2} d x\right) \frac{\partial^{2} u}{\partial x^{2}}=0,
$$

presented by Kirchhoff in 1883 (see [10]). This equation is an extension of the classical d'Alembert's wave equation by considering the effect of the changes in the length of the string during the vibrations. The parameters in (1.2) have the following meanings: $L$ is the length of the string, $h$ is the area of the cross-section, $E$ is the Young modulus of the material, $\rho$ is the mass density, and $P_{0}$ is the initial tension.

In recent years, problems involving Kirchhoff type operators have been studied in many papers, we refer to $([3-5,7,9,11])$, in which the authors have used different methods to get the existence of solutions for Kirchhoff type equations. Our paper is motivated by the recent results in $([1,2])$. In the paper [2], Azzouz and Bensedik studied the existence of a positive weak solution for the nonlocal problem of the form

$$
\left\{\begin{array}{l}
-M\left(\int_{\Omega}|\nabla u|^{2} d x\right) \Delta u=|u|^{p-2} u+\lambda f(x) \text { in } \Omega, \\
u=0 \text { on } \partial \Omega
\end{array}\right.
$$

where $\Omega$ is a bounded smooth domain in $\mathbb{R}^{N}, N \geq 3$ and $p>1$, with a sign-changing function $f$.

Using the sub-supersolution method combining a comparison principle introduced in [1], the authors established the existence of a positive solution for (1.3), where the parameter $\lambda>0$ is small enough. In the present paper, we consider system (1.1) in the case when the nonlinearities are "sublinear" at infinity, see the condition ( $H$ 3). Under suitable conditions on $f, g, h$ and $\tau$, we shall show that system (1.1) has a positive solution for $\lambda>\lambda^{*}$ large enough. To our best knowledge, this is a new research topic for nonlocal problems, see [8]. In current paper, motivated by previous works in $([2],[6])$ and by using sub-super solutions method, we study of existence of weak positive solutions for a new class of Kirrchoff elliptic systems in bounded domains with multiple parameters, where the right hand side defined as a multiplication of two separate functions. Our results extend and improve our recent results in [3] and [11].

\section{Existence Result}

Lemma $2.1([2])$. Assume that $M: \mathbb{R}^{+} \rightarrow \mathbb{R}^{+}$is a continuous and nonincreasing function satisfying $\lim _{t \rightarrow 0^{+}} M(t)=m_{0}$, where $m_{0}$ is a positive constant. Suppose further that function $H(t):=t M\left(t^{2}\right)$ is increasing on $\mathbb{R}$. 
Assume that $u, v$ are two non-negative functions such that

$$
\left\{\begin{array}{l}
-M\left(\int_{\Omega}|\nabla u|^{2} d x\right) \Delta u \geq-M\left(\int_{\Omega}|\nabla v|^{2} d x\right) \triangle v \text { in } \Omega \\
u=v=0 \text { on } \partial \Omega
\end{array}\right.
$$

then $u \geq v$ a.e. in $\Omega$.

Lemma 2.2 ([1]). If $M$ verifies the conditions of Lemma 2.1, then for each $f \in L^{2}(\Omega)$ there exists a unique solution $u \in H_{0}^{1}(\Omega)$ to the $M$-linear problem

$$
\left\{\begin{array}{l}
-M\left(\int_{\Omega}|\nabla u|^{2} d x\right) \triangle u=f(x) \text { in } \Omega, \\
u=0 \text { in } \partial \Omega .
\end{array}\right.
$$

Lemma $2.3([6])$. Let $w$ solve $\Delta w=g$ in $\Omega$. If $g \in C(\Omega)$, then $w \in C^{1, \alpha}(\Omega)$ for any $\alpha \in(0,1)$, so particularly $w$ is continuous in $\Omega$.

In this section, we shall state and prove the main result of this paper. Let us assume the following assumptions.

(H1) Assume that $A, B: \mathbb{R}^{+} \rightarrow \mathbb{R}^{+}$satisfy the same conditions as $M$ in Lemma 1 , and there exists $a_{i}, b_{i}>0, i=1,2$, such that

$$
a_{1} \leq A(t) \leq a_{2}, \quad b_{1} \leq B(t) \leq b_{2}, \quad \text { for all } t \in \mathbb{R}^{+} .
$$

(H2) $\alpha, \beta \in C(\bar{\Omega})$ and

$$
\alpha(x) \geq \alpha_{0}>0, \quad \beta(x) \geq \beta_{0}>0,
$$

for all $x \in \Omega$.

(H3) $f, g, h$, and $\tau$ are $C^{1}$ on $(0,+\infty)$, and increasing functions such that

$\lim _{t \rightarrow+\infty} f(t)=+\infty, \quad \lim _{t \rightarrow+\infty} g(t)=+\infty, \quad \lim _{t \rightarrow+\infty} h(t)=+\infty=\lim _{t \rightarrow+\infty} \tau(t)=+\infty$.

(H4) Exists $\gamma>0$ such that

$$
\lim _{t \rightarrow+\infty} \frac{h(t) f\left(k\left[g(t)^{\gamma}\right]\right)}{t}=0, \quad \text { for all } k>0
$$

and

$$
\lim _{t \rightarrow+\infty} \frac{\tau\left(k t^{\gamma}\right)}{t^{\gamma-1}}=0, \quad \text { for all } k>0 .
$$

We present below an example where hypotheses $(H 3)$ and $(H 4)$ hold

$$
\tau(t)=\ln (t), \quad h(t)=\sqrt{t}, \quad f(t)=\ln (t), \quad g(t)=t, \quad \gamma=2 .
$$

Theorem 2.1. Assume that the conditions (H1)-(H4) hold. Then for $\lambda_{1} \alpha_{0}$ and $\lambda_{2} \beta_{0}$ large the problem (1.1) has a large positive weak solution.

We give the following two definitions before we give our main result. 
Definition 2.1. Let $(u, v) \in\left(H_{0}^{1}(\Omega) \times H_{0}^{1}(\Omega)\right),(u, v)$ is said to be a weak solution of (1.1) if it satisfies

$$
\begin{aligned}
& A\left(\int_{\Omega}|\nabla u|^{2} \mathrm{~d} x\right) \int_{\Omega} \nabla u \nabla \phi \mathrm{d} x=\lambda_{1} \int_{\Omega} \alpha(x) f(v) h(u) \phi \mathrm{d} x \text { in } \Omega, \\
& B\left(\int_{\Omega}|\nabla v|^{2} \mathrm{~d} x\right) \int_{\Omega} \nabla v \nabla \psi \mathrm{d} x=\lambda_{2} \int_{\Omega} \beta(x) g(u) \tau(v) \psi \mathrm{d} x \text { in } \Omega,
\end{aligned}
$$

for all $(\phi, \psi) \in\left(H_{0}^{1}(\Omega) \times H_{0}^{1}(\Omega)\right)$.

Definition 2.2. A pair of nonnegative functions $(\underline{u}, \underline{v}),(\bar{u}, \bar{v})$ in $\left(H_{0}^{1}(\Omega) \times H_{0}^{1}(\Omega)\right)$ are called a weak subsolution and supersolution of $(1.1)$ if they satisfy $(\underline{u}, \underline{v}),(\bar{u}, \bar{v})=$ $(0,0)$ on $\partial \Omega$

$$
\begin{aligned}
& A\left(\int_{\Omega}|\nabla \underline{u}|^{2} d x\right) \int_{\Omega} \nabla \underline{u} \nabla \phi \mathrm{d} x \leq \lambda_{1} \int_{\Omega} \alpha(x) f(\underline{v}) h(\underline{u}) \phi \mathrm{d} x \text { in } \Omega, \\
& B\left(\int_{\Omega}|\nabla \underline{v}|^{2} d x\right) \int_{\Omega} \nabla \underline{v} \nabla \psi \mathrm{d} x \leq \lambda_{2} \int_{\Omega} \beta(x) g(\underline{u}) \tau(\underline{v}) \psi \mathrm{d} x \text { in } \Omega
\end{aligned}
$$

and

$$
\begin{aligned}
& A\left(\int_{\Omega}|\nabla \bar{u}|^{2} d x\right) \int_{\Omega} \nabla \bar{u} \nabla \phi \mathrm{d} x \geq \lambda_{1} \int_{\Omega} \alpha(x) f(\bar{v}) h(\bar{u}) \phi \mathrm{d} x \text { in } \Omega, \\
& B\left(\int_{\Omega}|\nabla \bar{v}|^{2} d x\right) \int_{\Omega} \nabla \bar{v} \nabla \psi \mathrm{d} x \geq \lambda_{2} \int_{\Omega} \beta(x) g(\bar{u}) \tau(\bar{v}) \psi \mathrm{d} x \text { in } \Omega,
\end{aligned}
$$

for all $(\phi, \psi) \in\left(H_{0}^{1}(\Omega) \times H_{0}^{1}(\Omega)\right)$.

Proof of Theorem 1. Let $\sigma$ be the first eigenvalue of $-\triangle$ with Dirichlet boundary conditions and $\phi_{1}$ the corresponding positive eigenfunction, with $\left\|\phi_{1}\right\|=1$. Let $m_{0}, \delta>$ 0 be such that $\left|\nabla \phi_{1}\right|^{2}-\sigma \phi_{1}^{2} \geq m_{0}$ on $\bar{\Omega}_{\delta}=\{x \in \Omega: d(x, \partial \Omega) \leq \delta\}$.

For each $\lambda_{1} \alpha_{0}$ and $\lambda_{2} \beta_{0}$ large, let us define

$$
\underline{u}=\left(\frac{\lambda_{1} \alpha_{0}}{2 a_{1}}\right) \phi_{1}^{2} \quad \text { and } \quad \underline{v}=\left(\frac{\lambda_{2} \beta_{0}}{2 b_{1}}\right) \phi_{1}^{2},
$$

where $a_{1}, b_{1}$ are given by the condition $(H 1)$. We shall verify that $(\underline{u}, \underline{v})$ is a weak subsolution of problem (1.1), for $\lambda_{1} \alpha_{0}$ and $\lambda_{2} \beta_{0}$ large enough. Indeed, let $\phi \in H_{0}^{1}(\Omega)$ with $\phi \geq 0$ in $\Omega$. By $(H 1)-(H 3)$, a simple calculation shows that

$$
A\left(\int_{\bar{\Omega}_{\delta}}|\nabla \underline{u}|^{2} d x\right) \int_{\bar{\Omega}_{\delta}} \nabla \underline{u} . \nabla \phi d x=A\left(\int_{\bar{\Omega}_{\delta}}|\nabla \underline{u}|^{2} d x\right) \frac{\lambda_{1} \alpha_{0}}{a_{1}} \int_{\bar{\Omega}_{\delta}} \phi_{1} \nabla \phi_{1} . \nabla \phi d x
$$




$$
\begin{aligned}
= & \frac{\lambda_{1} \alpha_{0}}{a_{1}} A\left(\int_{\bar{\Omega}_{\delta}}|\nabla \underline{u}|^{2} d x\right) \\
& \times\left\{\int_{\bar{\Omega}_{\delta}} \nabla \phi_{1} \nabla\left(\phi_{1} . \phi\right) d x-\int_{\bar{\Omega}_{\delta}}\left|\nabla \phi_{1}\right|^{2} \phi d x\right\} \\
= & \frac{\lambda_{1} \alpha_{0}}{a_{1}} A\left(\int_{\bar{\Omega}_{\delta}}|\nabla \underline{u}|^{2} d x\right) \int_{\bar{\Omega}_{\delta}}\left(\sigma \phi_{1}^{2}-\left|\nabla \phi_{1}\right|^{2}\right) \phi d x .
\end{aligned}
$$

On $\bar{\Omega}_{\delta}$ we have $\left|\nabla \phi_{1}\right|^{2}-\sigma \phi_{1}^{2} \geq m_{0}$, then $\sigma \phi_{1}^{2}-\left|\nabla \phi_{1}\right|^{2}<0$. So,

$$
A\left(\int_{\Omega_{\delta}}|\nabla \underline{u}|^{2} d x\right) \int_{\bar{\Omega}_{\delta}} \nabla \underline{u} \nabla \phi d x<0,
$$

by $(H 3)$ for $\lambda_{1} \alpha_{0}$ and $\lambda_{2} \beta_{0}$ large enough we get $f(\underline{v}) h(\underline{u})>0$. And then

$$
A\left(\int_{\bar{\Omega}_{\delta}}|\nabla \underline{u}|^{2} d x\right) \int_{\bar{\Omega}_{\delta}} \nabla \underline{u} \nabla \phi d x \leq \lambda_{1} \int_{\bar{\Omega}_{\delta}} \alpha(x) f(\underline{v}) h(\underline{u}) \phi d x .
$$

Next, on $\Omega \backslash \bar{\Omega}_{\delta}$ we have $\phi_{1} \geq r$ for some $r>0$, and therefore, by the conditions (H1)-(H3) and the definition of $\underline{u}$ and $\underline{v}$, it follows that

$$
\begin{aligned}
\lambda_{1} \int_{\Omega \backslash \bar{\Omega}_{\delta}} \alpha(x) f(\underline{v}) h(\underline{u}) \phi d x & \geq \frac{\lambda_{1} \alpha_{0} a_{2}}{a_{1}} \sigma \int_{\Omega \backslash \bar{\Omega}_{\delta}} \phi d x \\
& \geq \frac{\lambda_{1} \alpha_{0}}{a_{1}} A\left(\int_{\Omega \backslash \bar{\Omega}_{\delta}}|\nabla \underline{u}|^{2} d x\right) \int_{\Omega \backslash \bar{\Omega}_{\delta}} \sigma \phi d x \\
& \geq \frac{\lambda_{1} \alpha_{0}}{a_{1}} A\left(\int_{\Omega \backslash \bar{\Omega}_{\delta}}|\nabla \underline{u}|^{2} d x\right) \int_{\Omega \backslash \bar{\Omega}_{\delta}}\left(\sigma \phi_{1}^{2}-\left|\nabla \phi_{1}\right|^{2}\right) \phi d x \\
& =A\left(\int_{\Omega \backslash \bar{\Omega}_{\delta}}|\nabla \underline{u}|^{2} d x\right)_{\Omega \backslash \bar{\Omega}_{\delta}} \nabla \underline{u} \nabla \phi d x
\end{aligned}
$$

for $\lambda_{1} \alpha_{0}>0$ large enough.

Relations (2.1) and (2.2) imply that

$$
A\left(\int_{\Omega}|\nabla \underline{u}|^{2} d x\right) \int_{\Omega} \nabla \underline{u} \nabla \phi d x \leq \lambda_{1} \int_{\Omega} \alpha(x) f(\underline{v}) h(\underline{u}) \phi d x \text { in } \Omega,
$$

for $\lambda_{1} \alpha_{0}>0$ large enough and any $\phi \in H_{0}^{1}(\Omega)$, with $\phi \geq 0$ in $\Omega$. 
Similarly,

$$
B\left(\int_{\Omega}|\nabla \underline{v}|^{2} d x\right) \int_{\Omega} \nabla \underline{v} \nabla \psi d x \leq \lambda_{2} \int_{\Omega} \beta(x) g(\underline{u}) \tau(\underline{v}) \psi d x \text { in } \Omega,
$$

for $\lambda_{2} \beta_{0}>0$ large enough and any $\psi \in H_{0}^{1}(\Omega)$, with $\psi \geq 0$ in $\Omega$. From (2.3) and (2.4), $(\underline{u}, \underline{v})$ is a subsolution of problem (1.1). Moreover, we have $\underline{u}>0$ and $\underline{v}>0$ in $\Omega, \underline{u} \rightarrow+\infty$ and $\underline{v} \rightarrow+\infty$ as $\lambda_{1} \alpha_{0} \rightarrow+\infty$ and $\lambda_{2} \beta_{0} \rightarrow+\infty$.

Next, we shall construct a weak supersolution of problem (1.1). Let $e$ be the solution of the following problem

$$
\left\{\begin{array}{c}
-\triangle e=1 \text { in } \Omega \\
e=0 \text { on } \partial \Omega .
\end{array}\right.
$$

Let

$$
\bar{u}=C e, \quad \bar{v}=\left(\frac{\lambda_{2}\|\beta\|_{\infty}}{b_{1}}\right)\left[g\left(C\|e\|_{\infty}\right)\right]^{\gamma} e,
$$

where $\gamma$ is given by $\left(H_{4}\right)$ and $C>0$ is a large positive real number to be chosen later. We shall verify that $(\bar{u}, \bar{v})$ is a supersolution of problem (1.1). Let $\phi \in H_{0}^{1}(\Omega)$ with $\phi \geq 0$ in $\Omega$. Then we obtain from (2.5) and the condition $(H 1)$ that

$$
\begin{aligned}
A\left(\int_{\Omega}|\nabla \bar{u}|^{2} d x\right) \int_{\Omega} \nabla \bar{u} . \nabla \phi d x & =A\left(\int_{\Omega}|\nabla \bar{u}|^{2} d x\right) C \int_{\Omega} \nabla e . \nabla \phi d x \\
& =A\left(\int_{\Omega}|\nabla \bar{u}|^{2} d x\right) C \int_{\Omega} \phi d x \\
& \geq a_{1} C \int_{\Omega} \phi d x .
\end{aligned}
$$

By $(H 4)$, we can choose $C$ large enough so that

$$
a_{1} C \geq \lambda_{1}\|\alpha\|_{\infty} f\left(\frac{\lambda_{2}\|\beta\|_{\infty}}{b_{1}}\|e\|_{\infty}\left[g\left(C\|e\|_{\infty}\right)\right]^{\gamma}\right) h\left(C\|e\|_{\infty}\right) .
$$

Therefore,

$$
\begin{aligned}
& A\left(\int_{\Omega}|\nabla \bar{u}|^{2} d x\right) \int_{\Omega} \nabla \bar{u} \cdot \nabla \phi d x \\
\geq & \lambda_{1}\|\alpha\|_{\infty} f\left(\frac{\lambda_{2}\|\beta\|_{\infty}}{b_{1}}\|e\|_{\infty}\left[g\left(C\|e\|_{\infty}\right)\right]^{\gamma}\right) \cdot h\left(C\|e\|_{\infty}\right) \int_{\Omega} \phi d x \\
\geq & \lambda_{1} \int_{\Omega}\|\alpha\|_{\infty} f\left(\frac{\lambda_{2}\|\beta\|_{\infty}}{b_{1}}\|e\|_{\infty}\left[g\left(C\|e\|_{\infty}\right)\right]^{\gamma}\right) \cdot h\left(C\|e\|_{\infty}\right) \phi d x \\
\geq & \lambda_{1} \int_{\Omega} \alpha(x) f(\bar{v}) h(\bar{u}) \phi \mathrm{d} x .
\end{aligned}
$$


Also,

$$
B\left(\int_{\Omega}|\nabla \bar{v}|^{2} d x\right) \int_{\Omega} \nabla \bar{v} \nabla \psi d x \geq \lambda_{2}\|\beta\|_{\infty} \int_{\Omega}\left[g\left(C\|e\|_{\infty}\right)\right]^{\gamma} \psi d x .
$$

Again by $(H 4)$ for $C$ large enough we have

$$
\left[g\left(C\|e\|_{\infty}\right)\right]^{\gamma} \geq g\left(C\|e\|_{\infty}\right) \tau\left(\frac{\lambda_{2}\|\beta\|_{\infty}\|e\|_{\infty}}{b_{1}}\left[g\left(C\|e\|_{\infty}\right)\right]^{\gamma}\right) .
$$

From (2.7) and (2.8), we have

$$
B\left(\int_{\Omega}|\nabla \bar{v}|^{2} d x\right) \int_{\Omega} \nabla \bar{v} \nabla \psi d x \geq \lambda_{2} \int_{\Omega} \beta(x) g(\bar{u}) \tau(\bar{v}) \psi d x .
$$

From (2.6) and (2.9) we have $(\bar{u}, \bar{v})$ is a weak supersolution of problem (1.1), with $\underline{u} \leq \bar{u}$ and $\underline{v} \leq \bar{v}$ for $C$ large.

In order to obtain a weak solution of problem (1.1) we define the sequence

$$
\left\{\left(u_{n}, v_{n}\right)\right\} \subset E=\left(H_{0}^{1}(\Omega) \times H_{0}^{1}(\Omega)\right) \cap(C(\Omega) \times C(\Omega))
$$

as follows: $\left(u_{0}, v_{0}\right):=(\bar{u}, \bar{v}) \in E$ and $\left(u_{n}, v_{n}\right)$ is the unique solution of the system

$$
\left\{\begin{array}{l}
-A\left(\int_{\Omega}\left|\nabla u_{n}\right|^{2} d x\right) \triangle u_{n}=\lambda_{1} \alpha(x) f\left(v_{n-1}\right) h\left(u_{n-1}\right) \text { in } \Omega \\
-B\left(\int_{\Omega}\left|\nabla v_{n}\right|^{2} d x\right) \triangle v_{n}=\lambda_{2} \beta(x) g\left(u_{n-1}\right) \tau\left(v_{n-1}\right) \text { in } \Omega \\
u_{n}=v_{n}=0 \text { on } \partial \Omega .
\end{array}\right.
$$

Problem $(2.10)$ is $(A, B)$-linear in the sense that, if $\left(u_{n-1}, v_{n-1}\right) \in E$ is a given, the right hand sides of $(2.10)$ is independent of $u_{n}, v_{n}$.

Set $\widetilde{A}(t)=t A\left(t^{2}\right), \widetilde{B}(t)=t B\left(t^{2}\right)$. Then, since $\widetilde{A}(\mathbb{R})=\mathbb{R}, \widetilde{B}(\mathbb{R})=\mathbb{R}, f\left(v_{0}\right)$, $h\left(u_{0}\right), g\left(u_{0}\right)$ and $\tau\left(v_{0}\right) \in C(\Omega) \subset L^{2}(\Omega)$ (in $x$ ), we deduce from Lemma 2.2 that system $(2.10)$, with $n=1$ has a unique solution $\left(u_{1}, v_{1}\right) \in\left(H_{0}^{1}(\Omega) \times H_{0}^{1}(\Omega)\right)$. And by observing that

$$
\left\{\begin{array}{l}
-\triangle u_{1}=\frac{\lambda_{1}}{A\left(\int_{\Omega}\left|\nabla u_{1}\right|^{2} d x\right)} \alpha f\left(v_{0}\right) h\left(u_{0}\right) \in C(\Omega), \\
-\triangle v_{1}=\frac{\lambda_{2}}{B\left(\int_{\Omega}\left|\nabla v_{1}\right|^{2} d x\right)} \beta g\left(u_{0}\right) \tau\left(v_{0}\right) \in C(\Omega), \\
u_{1}=v_{1}=0 \text { on } \partial \Omega .
\end{array}\right.
$$


We deduce from Lemma 2.3 that $\left(u_{1}, v_{1}\right) \in C(\Omega) \times C(\Omega)$. Consequently $\left(u_{1}, v_{1}\right) \in E$. By the same way we construct the following elements $\left(u_{n}, v_{n}\right) \in E$ of our sequence. From $(2.10)$ and the fact that $\left(u_{0}, v_{0}\right)$ is a weak supersolution of $(1.1)$, we have

$$
\left\{\begin{array}{l}
-A\left(\int_{\Omega}\left|\nabla u_{0}\right|^{2} d x\right) \triangle u_{0} \geq \lambda_{1} \alpha(x) f\left(v_{0}\right) h\left(u_{0}\right)=-A\left(\int_{\Omega}\left|\nabla u_{1}\right|^{2} d x\right) \triangle u_{1}, \\
-B\left(\int_{\Omega}\left|\nabla v_{0}\right|^{2} d x\right) \triangle v_{0} \geq \lambda_{2} \beta(x) g\left(u_{0}\right) \tau\left(v_{0}\right)=-B\left(\int_{\Omega}\left|\nabla v_{1}\right| d x\right) \triangle v_{1},
\end{array}\right.
$$

and by Lemma $1, u_{0} \geq u_{1}$ and $v_{0} \geq v_{1}$. Also, since $u_{0} \geq \underline{u}, v_{0} \geq \underline{v}$ and the monotonicity of $f, h, g$, and $\tau$ one has

$$
\begin{aligned}
-A\left(\int_{\Omega}\left|\nabla u_{1}\right|^{2} d x\right) \triangle u_{1} & =\lambda_{1} \alpha(x) f\left(v_{0}\right) h\left(u_{0}\right) \\
& \geq \lambda_{1} \alpha(x) f(\underline{v}) h(\underline{u}) \geq-A\left(\int_{\Omega}|\nabla \underline{u}|^{2} d x\right) \triangle \underline{u}, \\
-B\left(\int_{\Omega}\left|\nabla v_{1}\right|^{2} d x\right) \triangle v_{1} & =\lambda_{2} \beta(x) g\left(u_{0}\right) \tau\left(v_{0}\right) \\
& \geq \lambda_{2} \beta(x) g(\underline{u}) \tau(\underline{v}) \geq-B\left(\int_{\Omega}|\nabla \underline{v}|^{2} d x\right) \triangle \underline{v},
\end{aligned}
$$

from which, according to Lemma $1, u_{1} \geq \underline{u}, v_{1} \geq \underline{v}$, for $u_{2}, v_{2}$ we write

$$
\begin{aligned}
-A\left(\int_{\Omega}\left|\nabla u_{1}\right|^{2} d x\right) \triangle u_{1} & =\lambda_{1} \alpha(x) f\left(v_{0}\right) h\left(u_{0}\right) \\
& \geq \lambda_{1} \alpha(x) f\left(v_{1}\right) h\left(u_{1}\right)=-A\left(\int_{\Omega}\left|\nabla u_{2}\right|^{2} d x\right) \triangle u_{2}, \\
-B\left(\int_{\Omega}\left|\nabla v_{1}\right| d x\right) \triangle v_{1} & =\lambda_{2} \beta(x) g\left(u_{0}\right) \tau\left(v_{0}\right) \\
& \geq \lambda_{2} \beta(x) g\left(u_{1}\right) \tau\left(v_{1}\right)=-B\left(\int_{\Omega}\left|\nabla v_{2}\right|^{2} d x\right) \triangle v_{2},
\end{aligned}
$$

and then $u_{1} \geq u_{2}, v_{1} \geq v_{2}$. Similarly, $u_{2} \geq \underline{u}$ and $v_{2} \geq \underline{v}$ because

$$
\begin{aligned}
-A\left(\int_{\Omega}\left|\nabla u_{2}\right|^{2} d x\right) \triangle u_{2} & =\lambda_{1} \alpha(x) f\left(v_{1}\right) h\left(u_{1}\right) \\
& \geq \lambda_{1} \alpha(x) f(\underline{v}) h(\underline{u}) \geq-A\left(\int_{\Omega}|\nabla \underline{u}|^{2} d x\right) \triangle \underline{u},
\end{aligned}
$$




$$
\begin{aligned}
-B\left(\int_{\Omega}\left|\nabla v_{2}\right|^{2} d x\right) \triangle v_{2} & =\lambda_{2} \beta(x) g\left(u_{1}\right) \tau\left(v_{1}\right) \\
& \geq \lambda_{2} \beta(x) g(\underline{u}) \tau(\underline{v}) \geq-B\left(\int_{\Omega}|\nabla \underline{v}|^{2} d x\right) \triangle \underline{v} .
\end{aligned}
$$

Repeating this argument we get a bounded monotone sequence $\left\{\left(u_{n}, v_{n}\right)\right\} \subset E$ satisfying

$$
\begin{aligned}
& \bar{u}=u_{0} \geq u_{1} \geq u_{2} \geq \cdots \geq u_{n} \geq \cdots \geq \underline{u}>0, \\
& \bar{v}=v_{0} \geq v_{1} \geq v_{2} \geq \cdots \geq v_{n} \geq \cdots \geq \underline{v}>0 .
\end{aligned}
$$

Using the continuity of the functions $f, h, g$, and $\tau$ and the definition of the sequences $\left\{u_{n}\right\},\left\{v_{n}\right\}$, there exist constants $C_{i}>0, i=1, \ldots, 4$, independent of $n$ such that

$$
\left|f\left(v_{n-1}\right)\right| \leq C_{1}, \quad\left|h\left(u_{n-1}\right)\right| \leq C_{2}, \quad\left|g\left(u_{n-1}\right)\right| \leq C_{3}
$$

and

$$
\left|\tau\left(u_{n-1}\right)\right| \leq C_{4}, \quad \text { for all } n .
$$

From (2.11), multiplying the first equation of (2.10) by $u_{n}$, integrating, using the Hölder inequality and Sobolev embedding we can show that

$$
\begin{aligned}
a_{1} \int_{\Omega}\left|\nabla u_{n}\right|^{2} d x & \leq A\left(\int_{\Omega}\left|\nabla u_{n}\right|^{2} d x\right) \int_{\Omega}\left|\nabla u_{n}\right|^{2} d x \\
& =\lambda_{1} \int_{\Omega} \alpha(x) f\left(v_{n-1}\right) h\left(u_{n-1}\right) u_{n} d x \\
& \leq \lambda_{1}\|\alpha\|_{\infty} \int_{\Omega}\left|f\left(v_{n-1}\right)\right| \cdot\left|h\left(u_{n-1}\right)\right| \cdot\left|u_{n}\right| d x \\
& \leq C_{1} C_{2}\|\alpha\|_{\infty} \lambda_{1} \int_{\Omega}\left|u_{n}\right| d x \\
& \leq C_{5}\left\|u_{n}\right\|_{H_{0}^{1}(\Omega)} .
\end{aligned}
$$

Then

$$
\left\|u_{n}\right\|_{H_{0}^{1}(\Omega)} \leq C_{5}, \quad \text { for all } n,
$$

where $C_{5}>0$ is a constant independent of $n$. Similarly, there exists $C_{6}>0$ independent of $n$ such that

$$
\left\|v_{n}\right\|_{H_{0}^{1}(\Omega)} \leq C_{6}, \quad \text { for all } n .
$$

From (2.12) and (2.13), we infer that $\left\{\left(u_{n}, v_{n}\right)\right\}$ has a subsequence which weakly converges in $H_{0}^{1}\left(\Omega, \mathbb{R}^{2}\right)$ to a limit $(u, v)$ with the properties $u \geq \underline{u}>0$ and $v \geq \underline{v}>0$. Being monotone and also using a standard regularity argument, $\left\{\left(u_{n}, v_{n}\right)\right\}$ converges itself to $(u, v)$. Now, letting $n \rightarrow+\infty$ in $(2.10)$, we deduce that $(u, v)$ is a positive weak solution of system (1.1). The proof of theorem is now completed. 
Acknowledgements. The authors would like to thank the referee(s) for a number of valuable suggestions regarding a previous version of this paper.

\section{REFERENCES}

[1] C. O. Alves and F. J. S. A.Correa, On existence of solutions for a class of problem involving a nonlinear operator, Comm. Appl. Nonlinear Anal. 8 (2001), 43-56.

[2] N. Azouz and A. Bensedik, Existence result for an elliptic equation of Kirchhoff type with changing sign data, Funkcial. Ekvac. 55 (2012), 55-66.

[3] S. Boulaaras and R. Guefaifia, Existence of positive weak solutions for a class of Kirrchoff elliptic systems with multiple parameters, Math. Methods Appl. Sci. 41 (2018), 5203-5210.

[4] S. Boulaaras and A. Allahem, Existence of positive solutions of nonlocal $p(x)$-Kirchhoff evolutionary systems via Sub-Super Solutions Concept, Symmetry 11 (2019), 1-11.

[5] S. Boulaaras, R. Guefaifia and S. Kabli, An asymptotic behavior of positive solutions for a new class of elliptic systems involving of $(p(x), q(x))$-Laplacian systems, Bol. Soc. Mat. Mex. 25 (2019), 145-162.

[6] D. D. Hai and R. Shivaji, An existence result on positive solutions for a class of p-Laplacian systems, Nonlinear Anal. 56 (2004), 1007-1010.

[7] R. Guefaifia and S. Boulaaras, Existence of positive radial solutions for $(p(x), q(x))$-Laplacian systems, Appl. Math. E-Notes 18 (2018), 209-218.

[8] X. Hanand G. Dai, On the sub-supersolution method for $p(x)$-Kirchhoff type equations, J. Inequal. Appl. 283 (2012), 1-11.

[9] M. Chen, On positive weak solutions for a class of quasilinear elliptic systems, Nonlinear Anal. 62 (2005), 751-756.

[10] G. Kirchhoff, Vorlesungen Uber Mathematische Physik, B.G. Teubner, Leipzig, 1983.

[11] Y. Bouizem, S. Boulaaras and B. Djebbar, Some existence results for an elliptic equation of Kirchhoff-type with changing sign data and a logarithmic nonlinearity, Math. Methods Appl. Sci. 42 (2019), 2465-2474.

${ }^{1}$ Department of Mathematics, Faculty of Mathematics and Informatics, University of Science and Technology of Oran Mohamed Boudiaf El Mnaouar, Bir El DJir, Oran, 31000 Algeria

Email address: bouizem@univ-usto.dz

${ }^{2}$ Department of Mathematics, College of Sciences and Arts, Al-Rass, Qassim University, Kingdom of Saudi Arabia

${ }^{3}$ Laboratory of Fundamental and Applied Mathematics of Oran (LMFaO), University of Oran 1, Ahmed Benbella. Algeria

Email address: S.Boularas@qu.edu.sa

Email address: saleh_boulaares@yahoo.fr

Email address: bachir.djebbar@univ-usto.dz 